\section{Efecto del}

\section{desperdicio de}

\section{una siderúrgica}

\section{en bases y subbases}

\author{
granulares
}

Kevista Ciencia e Ingeniería Neogranadina ISSN 01248170, ND 13 (Págs. 25-29)

\author{
ÓSCAR J. REYES ORTIZ* \\ JAVIER FERNANDO CAMACHO TAUTA \\ GRUPO INVESTIGACIÓN GEOTECNIA \\ UNIVERSIDAD MILITAR NUEVA GRANADA
}

\title{
Resumen
}

El artículo describe el objetivo, metodología, análisis y conclusiones de varios estudios experimentales realizados en los Laboratorios de la Universidad Militar Nueva Granada para determinar la viabilidad del empleo del desperdicio de alto horno generada por la siderúrgica de Boyacá en la mejora de las propiedades mecánicas de las bases y subbases granulares entpleadas para la construcción de una estructura de pavimentos, mediante la ejecución de los ensayos proctor estándar y modificado y C.B.R. de laboratorio.

La metodologia empleada para el desarrollo de las investigaciones se efectuó en primera fase con

\footnotetext{
Ingentero Cavil y Mogister in Ingenterin Covil de ta Universidod de los Andes. Especialista en finanzos de lo Universidod del Rasonio. Jefe del Ánea de Geotecrio de la Universidad Millitar Nueva Granada

- Ingenero Civn Universidad Militar Nueva Granada. Aagister an Ingenicra Cuvil de la Unrversidad de Los Audes. Dacente tiempo compjeto de lo Unuversidad Malitor Nueva Granado
} 
una caracterización del material granular y el material de desperdicio para emplear como reemplazo, posteriormente con la determinación de los porcentajes óptimos de humedad y resistencia del material virgen y con reemplazo, finalizando con los análisis y conclusiones.

Palabras clave: Estructura de pavimento, desperdicio de alto horno de una siderúrgica, ensayo proctor y C.B.R.

\section{INTRODUCCIÓN}

Los crecientes problemas ambiencales y energéticos del planeta se convierten en una variable primordial para la ejecución de cualquier obra civil. En el caso particular de la infraestructura vial el incremento de las cargas por eje en los vehiculos pesados, el desarrollo de la tecnología automotriz y la carencia de materiales granulares con propiedades mecánicas y fisicas acorde con las necesidades, han hecho que las instituciones de investigación adelanten procesos para desarrollar aditivos, los cuales generan o estabilizan las propiedades mecánicas de las capas que conforman la estructura de pavimento. Prueba de ello son las investigaciones sobre nateriales del tipo polímeros que se introducen en las capas asfálticas, el empleo de cemento o cal en las bases y subbases granulares y aceites sulfonados en las subrasances \{1\}.

Con la preocupación del impacto ambiental que generan algunos materiales en el ambiente, se desarrollaron diferentes investigaciones en los laboratorios de la Universidad Militar Nueva Granada, con el fin de decerminar la influencia que tiene el desperdicio generado en el proceso de la elaboración del acero en las capas de bases y subbases granulares que conforman una estructura de pavimento.

\section{MARCO TEÓRICO}

El desperdicio de la siderúrgica o escoria de cll. chara, se forma a partir de la fusión de impurczas de minerales agregados al proceso de producción de acero, con el fin de separar los óxidos como la alúmina y el sílice que pernite hacer la mezda necesaria para la elaboración del mismo. la extracción de la escoria líquida de cuchara se hace aproximadamente a $1400^{\circ} \mathrm{C}$, por ser esta la temperatura a la cual se hace la colada [2]. I:I enfriamiento puede ser rápido o lento dependiendo del proceso de extracción, se puede obtener escoria granulada con la caracteristica de endurecerse al contacto con el agua cuando su enfriamiento es rápido y escoria cristalizada si el enfriamiento es lento [3].

La escoria de aceración se separa durante y al final del proceso, obteniendo desperdicio con características diferentes tanto fisica como químicamente. La figura 1 muestra el diagrama de flujo de ta producción de la escoria en el proceso siderúrgico.

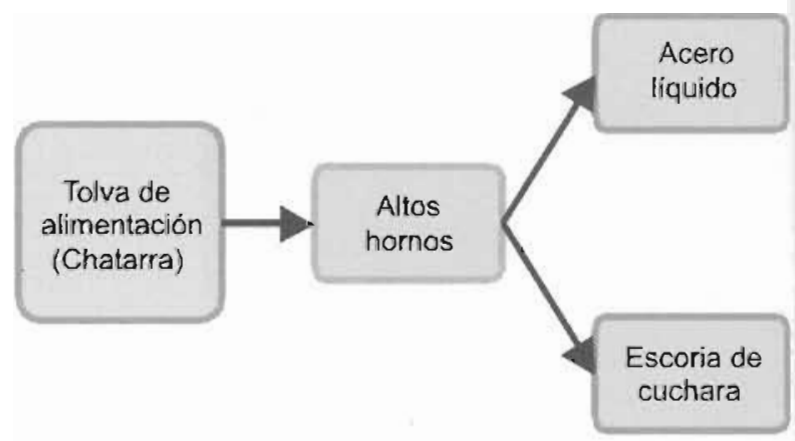

Figura No. 1. Proceso Sidenurgice

\section{METODOLOGÍA}

El desarrollo de las investigaciones experimentales en el laboratorio se realizó ell primera instancia con la caracterización del material granular con 
los ensayos de granulometría, contenido de materia orgánica, desgaste en la máquina de los Ángeles, equivalentes de arena, límites de Atterberg entre otros [4]. Posteriormente se determinaron las propiedades mecánicas y físicas del material para utilizar como reemplazo del granular (desperdicio de la siderúrgica).

Con las características mecánicas del material granular y las curvas granulométricas SBC (subbase granular) y $B G-1$ y $B G-2$ (Bases granular tipo 1 y tipo 2) [5], se procedió a realizar el ensayo proctor modificado a cada una para determinar la humedad óptima y con esta realizar los ensayos de CBR, cuyos resultados corresponden a la muestra patrón para todos los ensayos, posterior-mente se procedió a reemplazar material por desperdicio de los tamices No. 10, 40, 200 y fondo. Adicionalmente, se realizó el reemplazo como un porcentaje del peso total de las muestras (1.5. $2.5,3.5,5.0,6.5$ y $8.0 \%)$.

Para analizar de la incidencia del desperdicio en las propiedades del material granular, se calculó la variación porcentual de las propiedades con respecto a la muestra patrón sin inclusión de desperdicio.

\section{PROPIEDADES FÍSICAS, QUÍMICAS $Y$ MINERALOGICAS DE LAS ESCORIAS}

En la tabla No. 1 se encuentra la composición de la escoria de la Siderúrgica de Boyacá, empleada en el estudio.

La escoria se excrae de la colada a una temperatura de $1400{ }^{\circ} \mathrm{C}$, lo cual genera pérdida de cualquier contenido de materia orgánica. Esta perdida pudo ser comprobada en esta investigación por la deterninación del contenido orgánico elı suelos mediante pérdida por ignición. En la tabla No. 2, se tiene la granulometría de la escoria de la siderúigica de Boyacá, empleada en la investigación.

\begin{tabular}{|c|c|c|}
\hline \multicolumn{2}{|c|}{ Compuesto } & Porcentaje (\%) \\
\hline Oxido de calcio & $\mathrm{CaO}$ & 46.5 \\
\hline Oxido de si!ıcio & $\mathrm{SiO}_{2}$ & 19.6 \\
\hline Óxido de hierro & $\mathrm{FeO}$ & 0.7 \\
\hline $\begin{array}{c}\text { Óxido de } \\
\text { magnesio }\end{array}$ & $\mathrm{MgO}$ & 1.2 \\
\hline & $\mathrm{P}_{2} \mathrm{O}_{5}$ & 0.01 \\
\hline Azulre & $\mathrm{S}$ & 0.7 \\
\hline & $\mathrm{Al}_{2} \mathrm{O}_{3}$ & 2.15 \\
\hline
\end{tabular}

Toblo No. 1. Composición de la Escoria de la Siderurgia de Boynce

\begin{tabular}{|c|c|c|c|}
\hline Tamiz & $\%$ Retenido & $\%$ Ret. Acum. & $\%$ Pasa \\
\hline $11 / 2^{\prime \prime}$ & 0.00 & 0.00 & 100.00 \\
\hline $1 "$ & 3.20 & 3.20 & 96.80 \\
\hline $3 / 4^{\prime \prime}$ & 1.32 & 4.52 & 95.48 \\
\hline $3 / 8^{\prime \prime}$ & 9.30 & 13.82 & 86.18 \\
\hline 4 & 7.83 & 21.66 & 78.34 \\
\hline 10 & 12.18 & 33.84 & 66.16 \\
\hline 20 & 12.45 & 46.28 & 53.72 \\
\hline 40 & 11.01 & 57.30 & 42.70 \\
\hline 80 & 12.22 & 69.51 & 30.49 \\
\hline 100 & 13.63 & 83.14 & 16.86 \\
\hline 200 & 11.24 & 94.39 & 5.61 \\
\hline Pasa 200 & 5.61 & 100.00 & 0.00 \\
\hline
\end{tabular}

Tabla No. 2. Granulometria Escoria de ta Siderügica de Bayacú

\section{ANÁLISIS DE RESULTADOS}

En la figura No. 3 se presenta la variación en la densidad seca con la adición de desperdicio de alto homo para diferentes tipos de granulometria. Únicamente se presenta mejora entre 1 y $3 \%$ en la granulometría tipo BG-2 y esta mejoría aumenta cuando se reemplaza material por escoria de tamaño más grueso (tamaños retenidos tamiz No. 10). Adicionalmente se observa que para las granulometrías BG1 y SBG, existe un decremento acentuado de la densidad a medida que se reemplaza el macerial granular por desperdicio para las particulas más finas. 


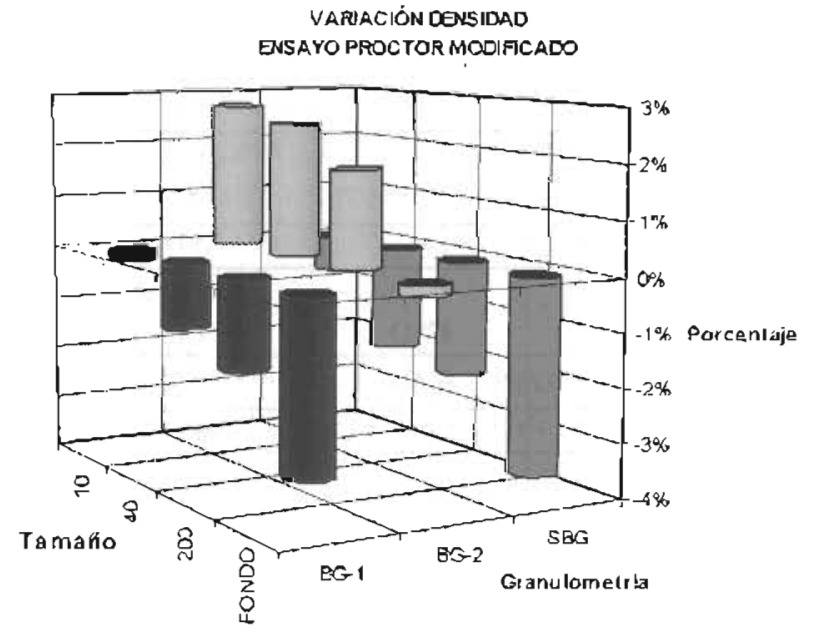

Figura No. 3. Variacion de la densidad seca

En la figura No. 4 se muestra la variación de la resistencia con el ensayo C.B.R. con la adición del desperdicio de alto horno para diferentes tipos de gramulometría. Se resalta que para subbases granulares se encontró que sin importar el marerial de reemplazo, siempre se presentaba incremenco de la resistencia. Siendo el óptimo para reemplazo del tamiz No. 10 y con un porcentaje de $18 \%$. Para la base granulares BG-2, todos los porcentajes determinaron incremento de la resistencia, su valor máximo (8\%) se obtuvo para el reemplazo con material pasa tamiz No. 200. Рага la base granular BG-1, los resultados no fueron satisfactorios. ya que no se presentaron incrementos significativos, hasta el punto de obtener un descenso para los reemplazos de pasa tamiz No. 10 y 200.

En la figura No. 5, se presenta la variación en la densidad seca y la resistencia con el ensayo C.B.R. a diferentes niveles de compactación con la adición de desperdicio de alto horno para la base granular tipo 2. Se observa que sin importar el porcentaje de escoria que se reemplace en la muestra, la densidad presenta incrementos que oscilan entre $0.22 \%$ y $3.6 \%$. De igual manera, se percibe que para niveles de compactación de 12

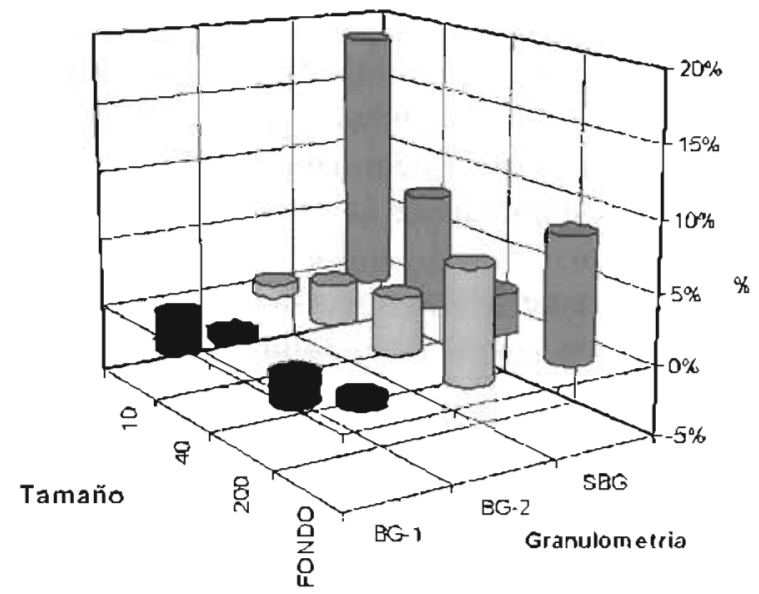

Figura No. 4. Variaciön de la resislencia (Ensayo C.B.R.)

y 26 golpes siempre existe un incremento de la resistencia y dicho valor oscila entre 80 y $260 \%$, sin embargo, para la compactación mayor (55 golpes, para porcentajes attos (8\%), se presenta un descenso de la resistencia en $23 \%$.

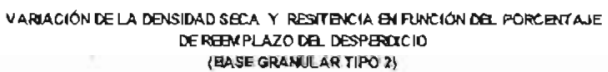
[EASE GRANULAR TIPO ?]

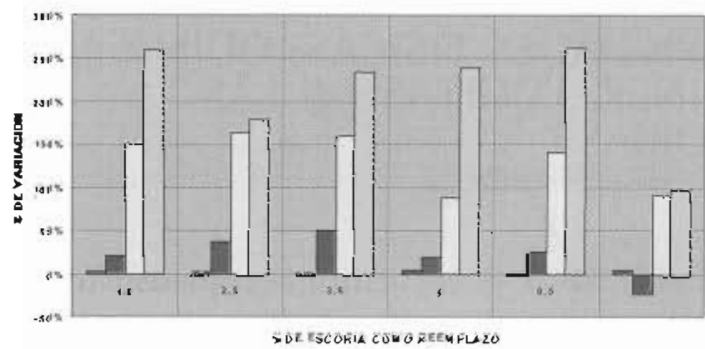

Figura No. 5. Veriacirin Densidad y C.B.R.

\section{CONCLUSIONES}

Mediante este proceso experimental se estudiaron diferentes opciones de mejoramiento o estabilización de las propiedades de las bases y subbases 
granulares según curvas granulométricas medias del Instituto Nacional de Vías, encontrando que al introducir desperdicio del alto horno de la Siderúrgica de Boyacá, se incrementó la densidad seca y la resistencia de las bases y subbases gramulares. De igual manera se pudo concluir que el cambiar materiales de diferentes tamaños (tamices No. 10, 40, 200 y fondo), se presentaba incremento de la densidad y la resistencia mediante el análisis de los ensayos de proctor y C.B.R. de laboratorio.

Por íltíno, se reemplazo por material de desperdicio de alto horno con respecto al peso total de las muestras de material de la granulometría media de base granular tipo 2, obteniendo incrementos en densidad y resistencia sin importar el nivel de compacración ni el porcentaje que varió entre el $1.5-8.0 \%$.

Su posible utilización lograría incrementar la resistencia y densidad de la estructura de pavimento. especialmente donde no se dispone de materiales de calidades óptimas o los métodos convencionales son de dificil ejecución.

Como recomendación final, es necesario continuar con este tipo de investigaciones, empleando diferentes rangos de dosificación, combinando dos 0 más materiales $y$ evaluando ofros parámetros que pueden intervenir en la resistencia y durabilidad de las soluciones propuestas.

\section{REFERENCIAS}

[1] Alcaldia Mayor Santa fe de Bogotá - IDU Uniandes. MANUAL DE DISEÑO DE PAVIMENTOS PARA SANTA FE DE BOGOTÁ. IDU. Bogotá. 2000.

[2] Fagua, Nestor. ESTABILIZACIÓN DE BASES Y SUBBASES CON LA ESCORIA DE LA SIDERÚRGICA DE BOYACÁ. Universidad Militar Nueva Granada. Bogotá. 2001.
[3| López, M. Lucrecia. USO DE ESCORIA PARA MEJORAR BASES Y SUBBASES GRANULARES. Universidad Militar Nueva Granada. Bogotá. 2002.

[4| Bowles, Joseph. MANUAL DE LABORATORIO DE SUELOS DE INGENIERİA CIVIL. Segunda edición. McGraw Hill. México. 1982.

[5| Instituto Nacional de Vías - INVIAS. NORMAS DE ENSAYO DE MATERIALES PARA CARRETERAS. Tomo 1 y II. INVIAS. Bogotá. 1998.

|6| Escorias siderúrgicas. MEMORIA TÉCNICA DE LAS PRIMERAS JORNADAS TÉCNICAS DE ESCORIA SIDERÚRGICAS. 1985.

[7] Reyes Lizcano. Fredy Alberto. DISEÑO RACIONAL DE PAVIMENTOS. Pontificia Universidad Javeriana. Bogotá. 2003. 